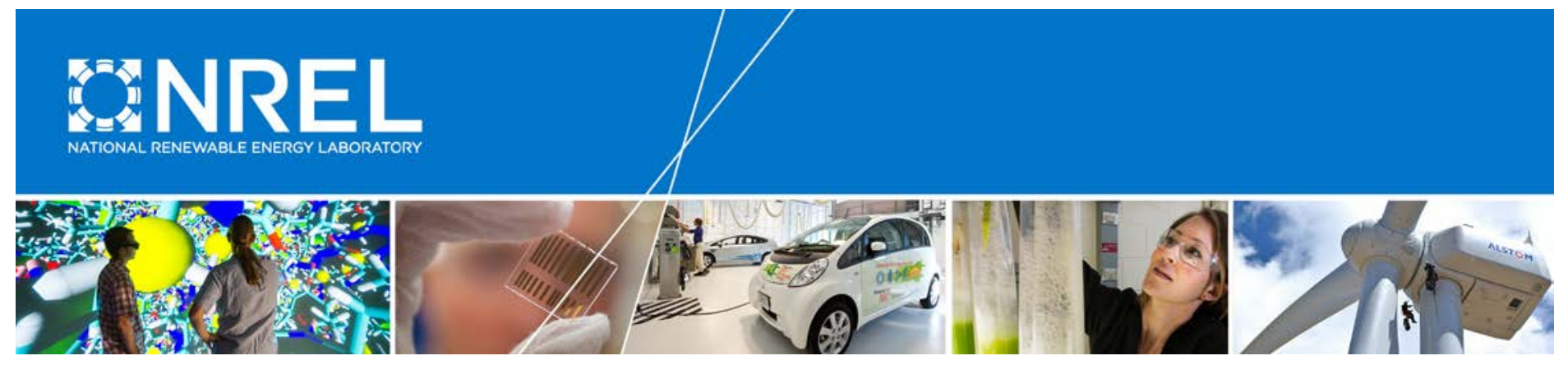

\title{
Rapid, Enhanced IV \\ Characterization of Multi- Junction PV Devices Under One Sun at NREL
}

\section{Preprint}

Tom Moriarty, Ryan France, and Myles Steiner National Renewable Energy Laboratory

Presented at the 42nd IEEE Photovoltaic Specialists Conference New Orleans, Louisiana

June 14-19, 2015

NREL is a national laboratory of the U.S. Department of Energy Office of Energy Efficiency \& Renewable Energy Operated by the Alliance for Sustainable Energy, LLC

This report is available at no cost from the National Renewable Energy Laboratory (NREL) at www.nrel.gov/publications.

\section{Conference Paper}

NREL/CP-5J00-64416

September 2015 


\section{NOTICE}

The submitted manuscript has been offered by an employee of the Alliance for Sustainable Energy, LLC (Alliance), a contractor of the US Government under Contract No. DE-AC36-08GO28308. Accordingly, the US Government and Alliance retain a nonexclusive royalty-free license to publish or reproduce the published form of this contribution, or allow others to do so, for US Government purposes.

This report was prepared as an account of work sponsored by an agency of the United States government. Neither the United States government nor any agency thereof, nor any of their employees, makes any warranty, express or implied, or assumes any legal liability or responsibility for the accuracy, completeness, or usefulness of any information, apparatus, product, or process disclosed, or represents that its use would not infringe privately owned rights. Reference herein to any specific commercial product, process, or service by trade name, trademark, manufacturer, or otherwise does not necessarily constitute or imply its endorsement, recommendation, or favoring by the United States government or any agency thereof. The views and opinions of authors expressed herein do not necessarily state or reflect those of the United States government or any agency thereof.

This report is available at no cost from the National Renewable Energy Laboratory (NREL) at www.nrel.gov/publications.

Available electronically at SciTech Connect http:/www.osti.gov/scitech

Available for a processing fee to U.S. Department of Energy and its contractors, in paper, from:

U.S. Department of Energy

Office of Scientific and Technical Information

P.O. Box 62

Oak Ridge, TN 37831-0062

OSTI http://www.osti.gov

Phone: 865.576.8401

Fax: 865.576.5728

Email: reports@osti.gov

Available for sale to the public, in paper, from:

U.S. Department of Commerce

National Technical Information Service

5301 Shawnee Road

Alexandria, VA 22312

NTIS http://www.ntis.gov

Phone: 800.553 .6847 or 703.605 .6000

Fax: 703.605.6900

Email: orders@ntis.gov 


\title{
Rapid, enhanced IV characterization of multi-junction PV devices under one sun at NREL
}

\author{
Tom Moriarty, Ryan France, Myles Steiner \\ National Renewable Energy Laboratory, Golden, CO 80401 USA
}

\begin{abstract}
Multi-junction technology is rapidly advancing, which puts increasing demands on IV characterization resources. We report on a tool and procedure for fast turn-around of IV data under the reference conditions, but also under controlled variations from the reference conditions. This enhanced data set can improve further iterations of device optimization.
\end{abstract}

\section{INTRODUCTION}

IV curves of PV devices under reference conditions are an essential metric for device performance and an essential tool for optimizing the design of succeeding generations of the particular technology. Conceptually, and in practice, this has been a straight forward process for single-junction devices through the use of the spectral mismatch correction factor method [1]. That is, use a reference cell with known QE, measure the QE of the test device, measure the spectrum of the simulator, and use these known quantities to calculate a spectral mismatch correction factor, M. This factor is used to adjust the total simulator irradiance, without changing its spectral shape, such that the test device will respond under the simulator spectrum as it would have responded under the reference spectrum.

This process is considerably more difficult and time consuming for multi-junction devices. A spectral mismatch correction factor must be calculated for each junction, and in general $\mathrm{M}$ will be different for each junction. Consequently, the spectral shape of the simulator must be adjusted as well at its total irradiance. It is usually very impractical to make multiple measurements under non-standard conditions because building the needed spectra tends to be very time consuming.

NREL's One-Sun Multi-Source Simulator (OSMSS) is a tool that greatly speeds this process. The OSMSS and the procedures used to build effective reference spectra have been previously described [2], and it has now been successfully used for up to six junction devices.

Here, we first present a process to vary the spectrum to one junction in a controlled fashion while keeping the effective irradiance to the remaining $\mathrm{n}-1$ junctions at the reference level. IV data collected under these circumstances is proving useful in optimizing new iterations of $4 \mathrm{~J}$ devices [3]. We also present IV data for a multi-junction device at several temperatures and irradiances. In this case the simulator spectrum was recreated for each temperature to provide the effective reference irradiance for the temperature varying QEs.

\section{BUILDING MODIFIED SPECTRA}

Let $\Phi_{\text {sim }}(\lambda)$ and $\Phi_{\text {ref }}(\lambda)$ be normalized simulator and reference spectra, and let $Q_{i}(\lambda)$ be the normalized QE for junction $\mathrm{i}$ of multi-junction device. If a test device is to behave under the simulator spectrum as it would under the reference spectrum then this is a necessary condition:

$$
\frac{\int \Phi_{R e f}(\lambda) Q_{i}(\lambda) d \lambda}{\int \Phi_{R e f}(\lambda) Q_{j}(\lambda) d \lambda}=\frac{\int \Phi_{\text {Sim }}(\lambda) Q_{i}(\lambda) d \lambda}{\int \Phi_{\text {Sim }}(\lambda) Q_{j}(\lambda) d \lambda}
$$

Define

$$
R_{i j}=\frac{\int \Phi_{R e f}(\lambda) Q_{i}(\lambda) d \lambda}{\int \Phi_{R e f}(\lambda) Q_{j}(\lambda) d \lambda} \times \frac{\int \Phi_{\text {Sim }}(\lambda) Q_{j}(\lambda) d \lambda}{\int \Phi_{\text {Sim }}(\lambda) Q_{i}(\lambda) d \lambda}
$$

Then the simulator spectrum shape will adequately reproduce the effect of the reference spectrum when $R_{i j}=1$ for all $i$ and $j$. A method for automated adjustment of the OSMSS spectrum to meet this condition is described in [2], but any method on any simulator that successfully sets $R_{i j}=1$ for all $i$ and $j$ will work.

Information on the behavior of individual junctions can be obtained by measuring IVs of the test device under various alternative conditions, such as temperature or spectrum. Alternative spectra can be treated as simple variations of the effective irradiance to each junction, $i$, by some factor, $\alpha_{i}$, Then in general, the test device will behave under the simulator spectrum as it would under the desired modified conditions when...

$$
\frac{\int\left[\alpha_{i} \Phi_{R e f}(\lambda, T)\right] Q_{i}(\lambda) d \lambda}{\int\left[\alpha_{j} \Phi_{R e f}(\lambda, T)\right] Q_{j}(\lambda) d \lambda}=\frac{\int \Phi_{S i m}(\lambda) Q_{i}(\lambda, T) d \lambda}{\int \Phi_{S i m}(\lambda) Q_{j}(\lambda, T) d \lambda}
$$

In which case...

$$
R_{i j}=\frac{\alpha_{i}}{\alpha_{j}} \frac{\int \Phi_{R e f}(\lambda, T) Q_{i}(\lambda) d \lambda}{\int \Phi_{R e f}(\lambda, T) Q_{j}(\lambda) d \lambda} \times \frac{\int \Phi_{S i m}(\lambda) Q_{j}(\lambda, T) d \lambda}{\int \Phi_{S i m}(\lambda) Q_{i}(\lambda, T) d \lambda}
$$

A useful approach to spectrum variation is to vary the irradiance to only one junction, say junction $k$. Then from (4), the desired spectrum will be achieved when $R_{k j}=\alpha_{k}$, $R_{i k}=\alpha_{k}{ }^{-1}$, and $R_{i j}=1$ for $i, j \neq k$.

The OSMSS automated spectrum building algorithm has been modified to build spectra with specified $R_{i j} \neq 1$. 


\section{VARIED SPECTRUM DATA EXAMPLE}

The NREL fabricated 4-junction inverted metamorphic (4JIMM) device, MN187, has been measured with the irradiance to each junction varied from 0.8 suns to 1.2 suns (AM1.5 Direct) while keeping the irradiance to the remaining three junctions at 1 sun. Fig. 1 shows the QEs of this 4-junction device overlaid with the OSMSS spectra that were built to satisfy $R_{1 j}=\alpha_{1}, R_{i 1}=\alpha_{k 1}{ }^{-1}$, and $R_{i j}=1$ for $i, j \neq 1$, where $\alpha_{1}$ varies from 0.8 to 1.2 . That is, the effective irradiance to the top junction was varied from 0.8 suns to 1.2 suns, while the effective irradiances to the other junctions were kept at one sun. This process was repeated for the other three junctions as well.

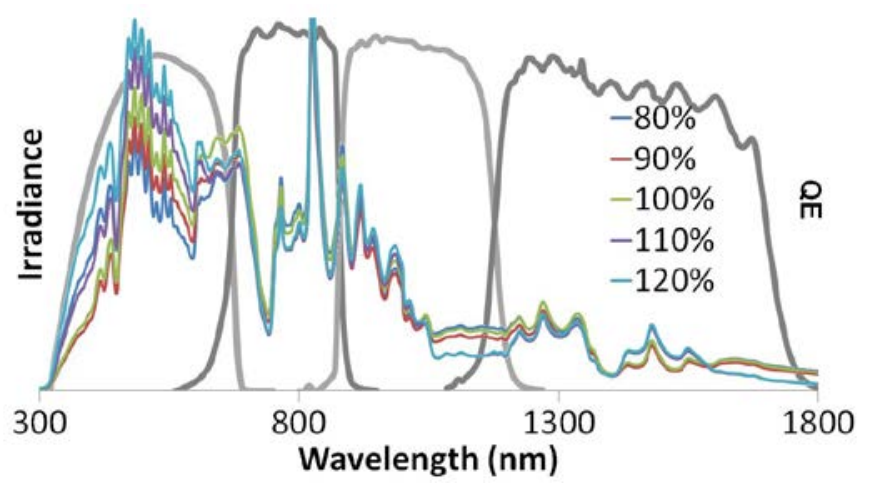

Figure 1. QEs for 4J-IMM and spectra built to vary effective irradiance to the top junction while keeping effective irradiance to junctions 2 through 4 at the effective reference irradiance.

Note that although the spectra in Fig 1 all provide the same effective irradiance to junctions 2 through 4 , they do not have exactly the same shape in the response range of those junctions. However, the criteria that $R_{1 j}=\alpha_{1}, R_{i 1}=\alpha_{k 1}{ }^{-1}$, and $R_{i j}=1$ are satisfied. There are an infinite number of ways to satisfy these criteria - it is only necessary to find one in the subset attainable by the simulator.

Figs 2, 3, 4, 5 \& 6 show the changes in Isc, Fill Factor, Vmax, Imax and Pmax, respectively resulting from the varied spectra.

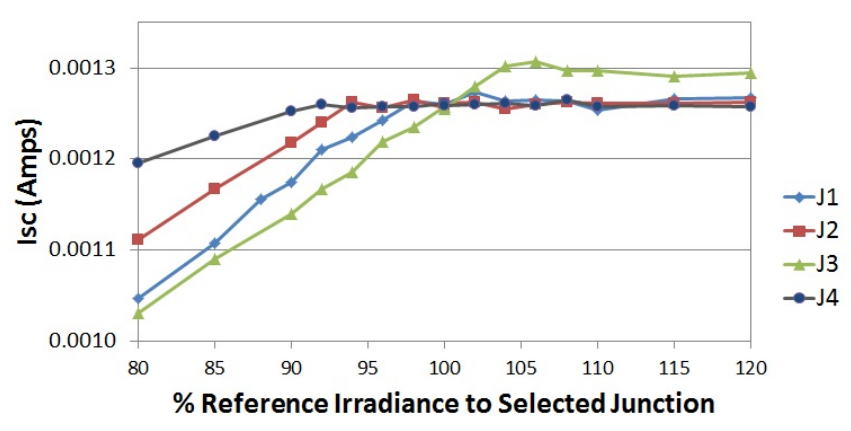

Figure 2. Isc vs. the percent of the effective reference irradiance to the selected junction. The effective reference irradiance to all other junctions is $100 \%$

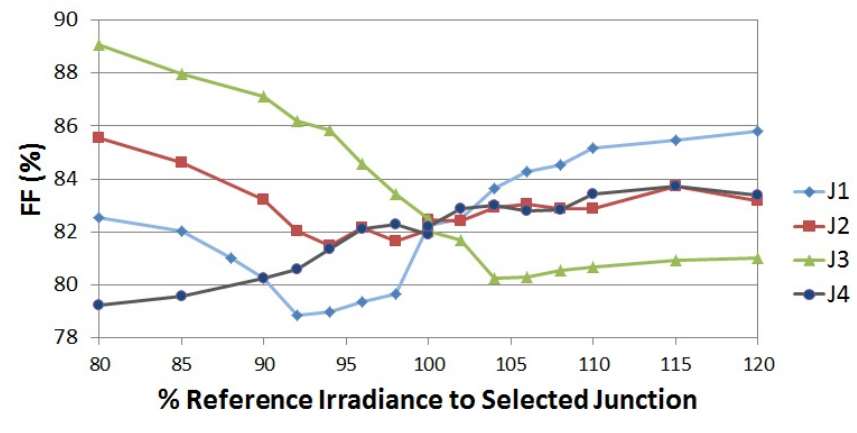

Figure 3. Isc vs. the percent of the effective reference irradiance to the selected junction. The effective reference irradiance to all other junctions is $100 \%$

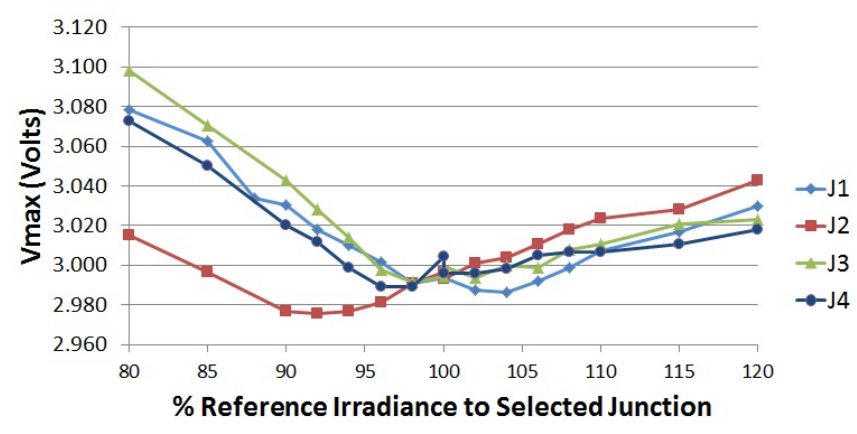

Figure 4. Vmax vs. the percent of the effective reference irradiance to the selected junction. The effective reference irradiance to all other junctions is $100 \%$.

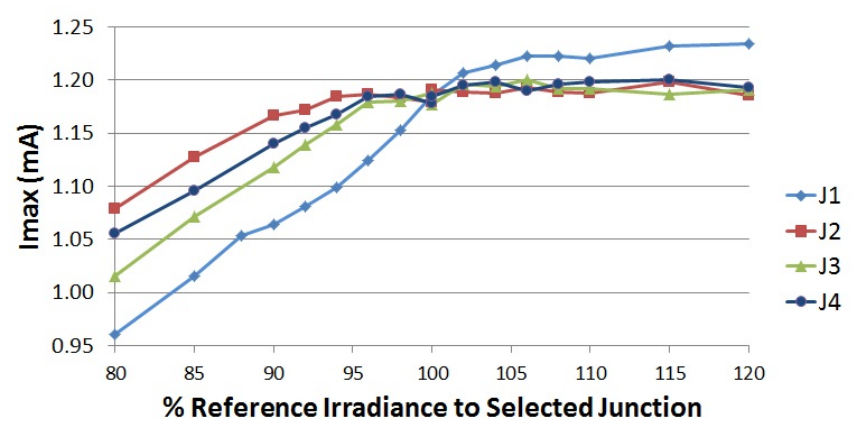

Figure 5. Imax vs. the percent of the effective reference irradiance to the selected junction. The effective reference irradiance to all other junctions is $100 \%$. 


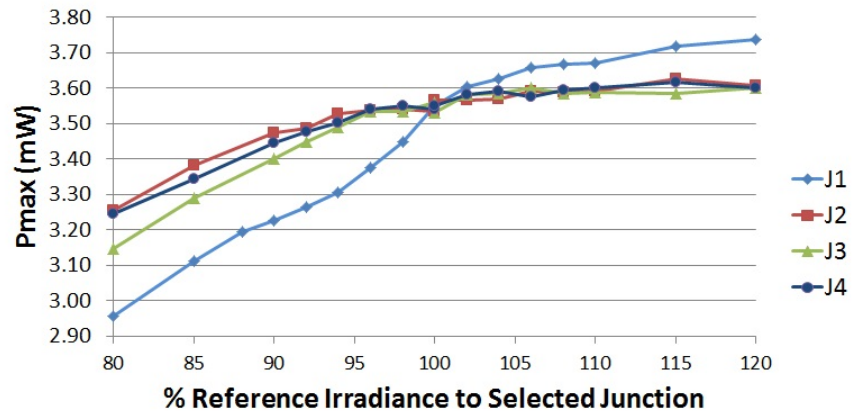

Figure 6. Pmax vs. the percent of the effective reference irradiance to the selected junction. The effective reference irradiance to all other junctions is $100 \%$.

\section{VARIED TEMPERATURE DATA EXAMPLE}

Measuring the IV response under the reference spectrum at varying temperatures is usually not a challenging undertaking for a single junction device with good thermal contact to a temperature controlled stage. It is considerably more complicated to properly make these measurements for a multjunction device because the temperature dependent QEs force a rebuild of the simulator's spectral shape for every temperature. NREL's traditional hardware and spectral mismatch correction method could require hours of set-up time to build a new spectrum at for each temperature for a three junction device. It was not practical for a device with more than three junctions and was never attempted.

This is now a straight forward and rapid process using the OSMSS. Fig 7 shows the QEs at three temperatures for a three junction GaInP/GaAs/GaInAs device (MH814) fabricated at NREL.

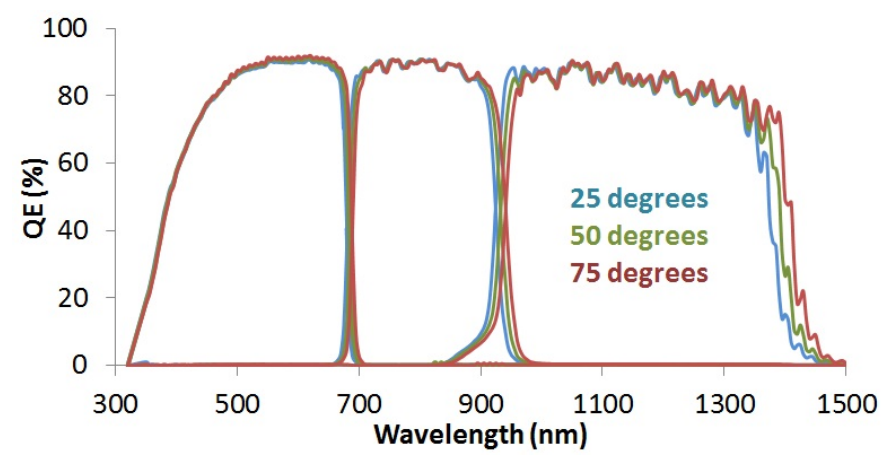

Figure 7. QEs for GaInP/GaAs/GaInAs (ID: MH814) at three temperatures
Fig 8 shows the resulting unscaled spectra that the OSMSS built to measure the IV of the device at the three temperatures under the effective AM1.5 global spectrum at $1000 \mathrm{~W} / \mathrm{m}^{2}$. Note the great variation of the spectra corresponding to the temperature changes. Nevertheless, the criteria that $R_{i j}=1$ for all $i$ and $j$ are satisfied.

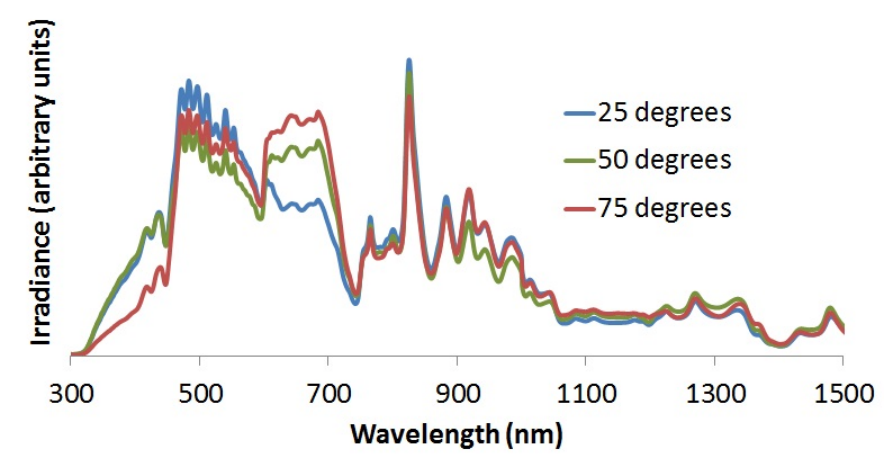

Figure 8 .

\section{CONCLUSION}

NREL's OSMSS's ability to rapidly build spectra opens up new IV characterization paths that were previously impractical. The data in Figs. 2 through 6 were collected in about three hours. Each data point in those figures represents an IV curve under simply defined, tightly controlled, unique irradiance conditions. Collecting the same set of data on this four junction device using NREL's traditional hardware and the spectral mismatch technique would have taken months - if it were possible at all.

Other possible research avenues include rapidly building representative spectra for simulator based yearly energy yield measurements. This should be possible by selecting the proper non-unity values for $\alpha_{i}$ and $\alpha_{j}$ in (4) to reproduce the effect of each spectrum.

\section{REFERENCES}

[1] C.R. Osterwald, "Translation of device performance measurements to reference conditions," Solar Cells, 18, pp 269279,1986

[2] T. Moriarty, J. Jablonski, K. Emery, "Algorithm for Building a Spectrum for NREL's One-Sun Multi-Source Simulator," Proceedings of the $38^{\text {th }}$ IEEE Photovoltaic Specialists Conference (PVSC), June 2012, pp1291-1295

[3] R. M. France, J. F. Geisz, I. García1, M. A. Steiner, W. E. McMahon, D. J. Friedman, T. E. Moriarty, C. Osterwald, J. S. Ward, A. Duda, M. Young, and W. J. Olavarria, "Design flexibility of 4-junction ulta-high efficiency inverted metamorphic solar cells", submitted to $42^{\text {nd }}$ IEEE PVSC, 2015. 